\title{
Recent advances in the chemistry and biology of anti- inflammatory and specialized pro-resolving mediators biosynthesized from n-3 docosapentaenoic acid
}

\author{
Anders Vik ${ }^{\mathrm{a},}$, Jesmond Dalli ${ }^{\mathrm{b}}$ and Trond Vidar Hansen ${ }^{\mathrm{a}}$ \\ ${ }^{a}$ School of Pharmacy, Department of Pharmaceutical Chemistry, University of Oslo, PO Box \\ 1068 Blindern, N-0316 Oslo, Norway \\ ${ }^{\mathrm{b}}$ William Harvey Research Institute, Barts and The London School of Medicine and Dentistry, \\ Queen Mary University of London, Charterhouse Square, London, UK, EC1M 6BQ
}

Email: anders.vik@ farmasi.uio.no

Phone: +4722857451

\begin{abstract}
Several novel oxygenated polyunsaturated lipid mediators biosynthesized from n-3 docosapentaenoic acid were recently isolated from murine inflammatory exudates and human primary cells. These compounds belong to a distinct family of specialized pro-resolving lipid mediators, and display potent in vivo anti-inflammatory and pro-resolution effects. The endogenously formed specialized pro-resolving mediators have attracted a great interest as lead compounds in drug discovery programs towards the development of new classes of drugs that dampen inflammation without interfering with the immune response. Detailed information on the chemical structures, cellular functions and distinct biosynthetic pathways of specialized pro-resolving lipid mediators is a central aspect of these efforts. Herein, the isolation, structural elucidation, biosynthetic pathways, total synthesis and bioactions of the n3 docosapentaenoic acid derived mediators PD $1_{n-3}$ DPA and MaR $1_{n-3}$ DPA are discussed. In addition, a brief discussion of a novel family of mediators derived from n-3 docosapentaenoic acid, termed 13-series resolvins is included.
\end{abstract}

Keywords: Inflammation; specialized pro-resolving lipid mediators; pro-resolution; $\mathrm{PD} 1_{\mathrm{n}-3}$ DPA; MaR1 $1_{n-3}$ DPA $; 13$-series resolvins; RvTs. 
Inflammation is divided into acute inflammation, which by nature is self-resolving, and chronic inflammation, which occurs over a longer period of time and does not resolve. ${ }^{1}$ Uncontrolled, excessive acute and chronic unresolved inflammation can result in the development of several human diseases, such as cardiovascular disease, cancer, rheumatoid arthritis, periodontal disease, asthma, diabetes, and inflammatory bowel disease, but also neurological disorders such as Alzheimer's disease and age related macular degeneration. ${ }^{2}$ The characteristics of acute inflammation include specific cellular events, such as increased permeability of endothelium cells leading to edema and infiltration of polymorphonuclear leukocytes, inflammatory macrophages and lymphocytes to the sites of infection or injury. Over the last century, inflammation has been the topic of numerous studies at the molecular and cellular level. ${ }^{3}$ These efforts have resulted in the identification of several chemical mediators, such as peptides, oxygenated polyunsaturated fatty acids (PUFAs), chemokines and cytokines, that initiate, modulate and reduce acute inflammatory processes. ${ }^{4}$ Today numerous drugs that dampen the effects of inflammatory processes have been developed. ${ }^{2}$ Until recently, resolution of inflammation was believed to be a passive process. However, the efforts headed by Professor Charles N. Serhan at Brigham \& Women's Hospital, Harvard Medical School, have established that the resolution phase of inflammation and the return to physiology (homeostasis) are regulated by actively and strictly controlled biosynthesis of several novel families of oxygenated PUFA derivatives. ${ }^{5}$ These endogenously formed compounds have been coined specialized pro-resolving mediators (SPMs). ${ }^{6}$ The lipoxins, derived from the n-6 PUFA arachidonic acid (AA), were the first family of SPMs to be reported. $^{7,8}$ The interesting biological activities of the lipoxins spurred an interest from the medicinal chemistry community in using the lipoxins as lead compounds in drug discovery programs. ${ }^{9-11}$ More recent studies have identified several novel SPMs biosynthesized from the dietary n-3 PUFAs eicosapentaenoic acid (EPA) and docosahexaenoic acid (DHA), see Figure 1. These di- and tri-hydroxy containing PUFA-derived lipid mediators are biosynthesized in the presence of cyclooxygenase and lipoxygenase enzymes during the resolution phase of acute inflammation. The E-series resolvins are derived from EPA, ${ }^{12}$ while the D-series resolvins, ${ }^{13}$ protectins ${ }^{13-15}$ and maresins ${ }^{16}$ as well as the recently described sulfido-conjugates RCTRs (resolvin conjugates in tissue regeneration), PCTRs (protectin conjugates in tissue regeneration), and MCTRs (maresin conjugates in tissue regeneration) are biosynthesized from DHA. ${ }^{17-25}$ 


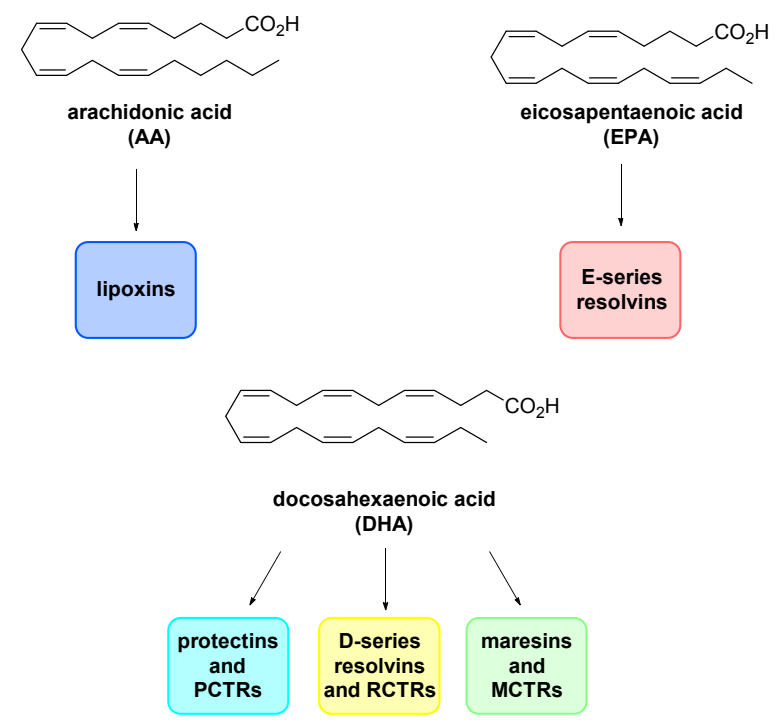

Figure 1. Overview of families of SPMs derived from AA, EPA and DHA.

The SPMs display a plethora of biological activities in promoting the termination of ongoing inflammation and regain of function. For example, SPMs limit further neutrophil recruitment to the inflammatory sites, accelerate the termination of inflammation resolution time in vivo and increase the clearance of bacteria and apoptotic cells, as reviewed by Serhan and Petasis. ${ }^{4}$ More recently, it has been reported that SPMs protect the host from bacterial infections ${ }^{26}$ and control tissue regeneration. ${ }^{17,27}$ These bioactions and the novel chemical structures of the compounds that the individual families of SPMs constitute, have attracted interest from the biomedical, pharmacological and synthetic organic communities. ${ }^{27}$ The SPMs display potent agonist effects in vivo, often in the low nanomolar range, acting on individual G-protein coupled receptors (GPCRs). ${ }^{28}$ For a summary of the current knowledge of SPM activities towards GPCRs, see recent reviews. ${ }^{29,30}$ In addition, several SPMs have entered clinical trial development programs. ${ }^{28}$ Recently, new SPMs biosynthesized from the PUFA n-3 docosapentaenoic acid (n-3 DPA) was reported ${ }^{31}$ (Figure 2). N-3 DPA is an elongated metabolite of EPA and is an intermediate in the biosynthesis of DHA. ${ }^{32}$ Several interesting biological effects have been reported for n-3 DPA. ${ }^{33}$ Herein, we will review the isolation, structural elucidation, biosynthetic pathways, total synthesis and bioactions of some of the SPMs derived from n-3 DPA. 


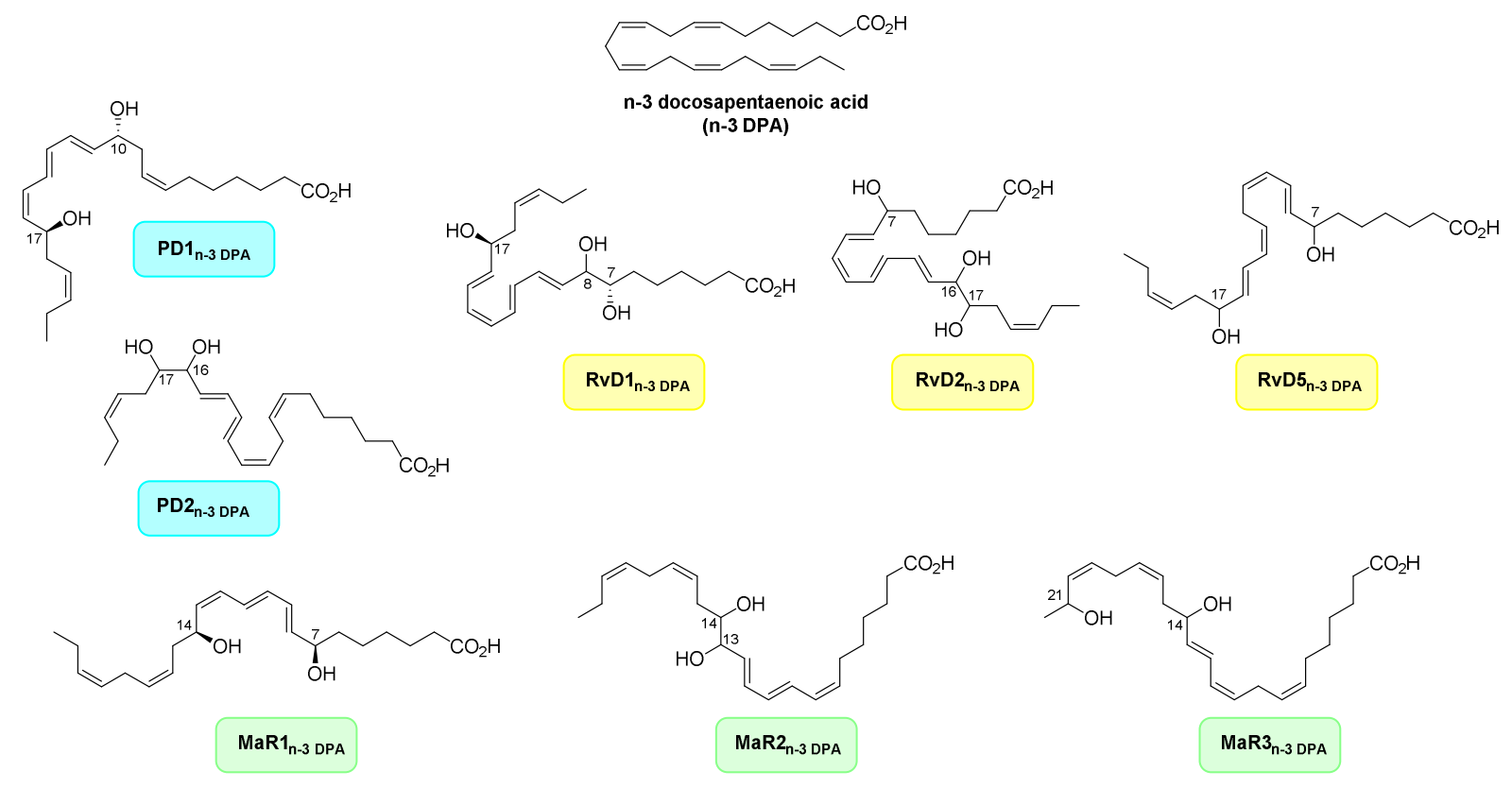

Figure 2. Chemical structures of n-3 DPA derived SPMs reported in $2013 .{ }^{31}$ Please note that in the original report, the absolute configurations were not reported for all SPMs. Where known, the absolute configurations have been included.

Isolation and structural elucidation of $P D 1_{n-3}{ }_{D P A}$ : The isolation and partial structural elucidation of n-3 docosapentaenoic acid derived protectin $1\left(P D 1_{n-3}\right.$ DPA $)$ was reported in 2013. ${ }^{31}$ The use of targeted LC/MS-MS metabololipidomics with material obtained during the onset and the resolution phase of acute inflammation revealed the presence of several tri- and di-hydroxy containing C22:5 PUFA compounds. Liquid chromatography analysis using UVmonitoring of material obtained from plasma of mice subjected to ischemia reperfusion injury and isolated human cells allowed the identification of five compounds. These were named $P D 1_{n-3}$ DPA,$P D 2_{n-3}$ DPA,$R v D 1_{n-3}$ DPA,$R v D 2_{n-3}$ DPA and RvD5 $5_{n-3}$ DPA, see Figure 2. UV/Vis spectroscopy, individual retention times and multiple reaction monitoring (MRM) chromatograms as well as structural elucidation of the fragment patterns in the MS-MS spectra, allowed the assignment of the chemical structure of PD1 $1_{n-3}$ DPA as 10,17dihydroxydocosa-7,11,13,15,19-pentaenoic acid. Of note, the configuration at C-17 was determined as $S$ using reverse phase chiral metabololipidomics analysis on material obtained from plasma isolated from mice subjected to ischemia reperfusion injury. The use of the two known mono-hydroxylated n-3 DPA derivatives $17 S$ - and 17R-HDPA for comparison of retention times with the novel compound, allowed the assignment of the $17 S$-configuration. 
These data also indicated the involvement of a mammalian lipoxygenase since this class of enzymes insert molecular oxygen in PUFAs predominantly in the $S$-configuration. ${ }^{34}$ However, the full stereochemical assignment of the double bond geometry in the conjugated triene system, as well as the absolute configuration of the C-10 hydroxyl group, was not assigned in the original report. The compounds depicted in Figure 2 were isolated in nano- to microgram amounts. Hence, total synthesis of PD1 $1_{n-3}$ DPA became necessary for establishing the complete configurational assignment. Direct comparison with synthetic material produced by total synthesis has been the strategy of choice for solving stereochemical issues of SPMs, as discussed below.

Biosynthesis of $P D 1_{n-3} D P A$ : A putative biosynthesis of PD1 $1_{n-3}$ DPA was disclosed in the original publication by Dalli et al. ${ }^{31}$ In this proposal, the PUFA n-3 DPA is first converted via 17 lipoxygenation to $17 S$-hydroperoxy-7Z,10Z,13Z,15E,19Z-docosapentaenoic acid (17S$\mathrm{H} p \mathrm{DPA}, \mathbf{1})$. Then $17 S-\mathrm{H} p \mathrm{DPA}(\mathbf{1})$ is converted into the intermediate epoxide $16,17 S$-epoxy$\mathrm{PD}_{\mathrm{n}-3}$ DPA (2) which is next enzymatically hydrolyzed to PD $1_{n-3}$ DPA and PD2 $2_{n-3}$ DPA, see Scheme 1. The detection and structural elucidation of four isomeric products observed after acidic methanol treatment supported the existence of $\mathbf{2}$ as an intermediate. Indirect evidence for the existence and involvement of the $16,17 S$-epoxy-PD $1_{n-3}$ DPA (2) intermediate is also provided from the biosynthesis of the structurally related protectin D1. ${ }^{35}$ Recently it was disclosed that synthetic $16 S, 17 S$-epoxy-PD1 was enzymatically converted to protectin D1 by human macrophages. ${ }^{36}$ It would be expected that the biosynthesis of the two SPMs PD1 and PD1 $1_{n-3}$ DPA would occur in a similar fashion, since both epoxides as well as SPMs are congeners. Hence, it is highly likely that the configuration is $S$ at C-16. The only structural difference between these two SPMs is the absence of the $4 Z$-double bond in PD $1_{n-3}$ DPA. This double bond does not participate in the biosynthesis of the two congeneric SPMs. However, conclusive evidence may only be obtained by preparing 2 by total synthesis and subject the synthetic material to biological systems. 


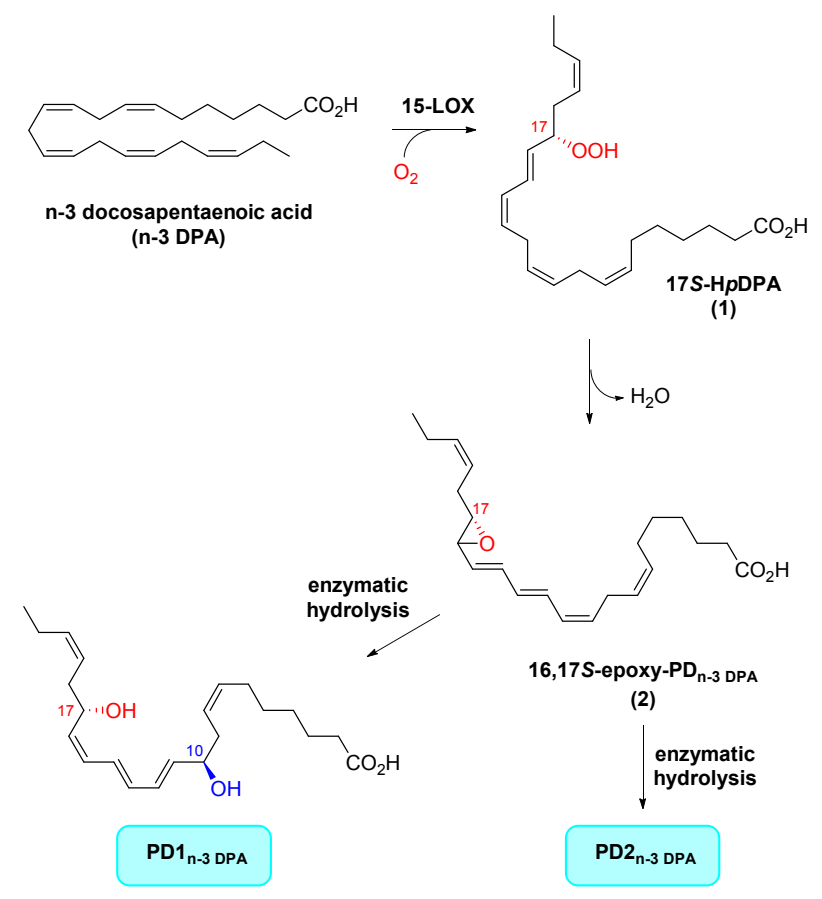

Scheme 1. Proposed biosynthesis of PD $1_{\mathrm{n}-3 \mathrm{DPA}}$. Note that the $S$-configuration is confirmed on C-17, but not at C-16.

Total synthesis of $P D 1_{n-3} D P A$ : Since PD1 $1_{\mathrm{n}-3 \mathrm{DPA}}$ as well as all of the other SPMs are produced in microgram to nanogram quantities in vivo, ${ }^{31}$ thorough biological evaluations and full configurational assignment depends on total synthesis of each SPM. Towards these aims, we initiated synthetic efforts towards developing a general strategy utilizing the knowledge and initial information on the SPM biosynthetic pathways. ${ }^{37}$ The two chiral secondary alcohols, present in numerous SPMs, are embedded by a $Z, E, E$-triene moiety. This triene system is a challenge to prepare in a high geometric purity. The highly functionalized aldehyde $(2 E, 4 E)$ 5-bromopenta-2,4-dienal (3) seemed well-suited for such purposes, ${ }^{38}$ in which both the vinylic bromide and the aldehyde are useful reaction partners (Scheme 2). The only reported total synthesis of PD1 $1_{n-3} \mathrm{DPA}^{39}$ commenced with the synthesis of the key intermediate 4 , that was prepared in excellent diastereomeric purity from commercially available salt 5. Pyridinium-1sulfonate (5) was treated with aqueous potassium hydroxide to yield potassium salt $\mathbf{6}$, which was first exposed to $\mathrm{PPh}_{3} / \mathrm{Br}_{2}$ in dichloromethane, and then $p$-TsOH in diethyl ether, to form (2E,4E)-5-bromopenta-2,4-dienal 3 in $75 \%$ yield over the two steps. The transformations of such quaternary pyridinium salts into useful functionalized conjugated dienes were recently reviewed. ${ }^{40}$ The aldehyde 3 was then reacted with thiazolidinone $7^{41}$ in an acetate-aldol reaction to produce intermediate $\mathbf{8}^{42,43}$ in a 15.3:1 diastereomeric ratio. Purification by chromatography yielded diastereomerically pure $\mathbf{8}$ in $86 \%$ yield. Next, protection of the 
secondary alcohol to give $\mathbf{9}$, followed by removal of the chiral auxiliary with DIBAL-H afforded aldehyde 4 .
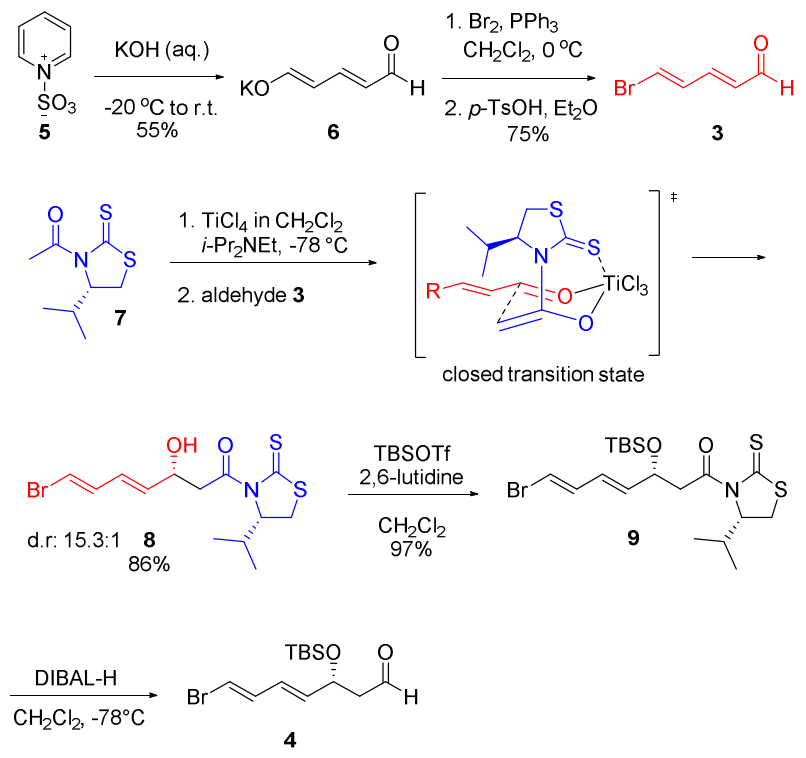

Scheme 2. Preparation of key intermediate 4 in the synthesis of $P D 1_{n-3}$ DPA.

Aldehyde 4 was then reacted immediately with the ylide of Wittig salt 10, prepared from cycloheptanone in four straightforward steps as outlined, in a Z-selective Wittig reaction to afford vinylic bromide 11 as one stereoisomer after purification (54\% yield from $\mathbf{9}$, Scheme 3 ). Under basic conditions, the aldehyde $\mathbf{4}$ is prone to elimination, resulting in an all conjugated system, hence the modest yield. A Sonogashira coupling with known acetylene $12,{ }^{44}$ prepared from $(R)$-glycidol and 1-propyne, and vinyl bromide 11 furnished the enyne $\mathbf{1 3}$ in $92 \%$ yield. Desilylation of the two protecting groups using TBAF in $\mathrm{THF}$ at $0{ }^{\circ} \mathrm{C}$ and subjecting the resulting diol to an atmosphere of hydrogen using the modified Lindlar conditions, ${ }^{45,46}$ afforded methyl ester 14 containing the light and heat sensitive E,E,Z-triene in high chemical and stereochemical purity in an acceptable yield. Finally, a basic hydrolysis of the methyl ester 14 afforded multi-milligrams of $P D 1_{n-3}$ DPA. 


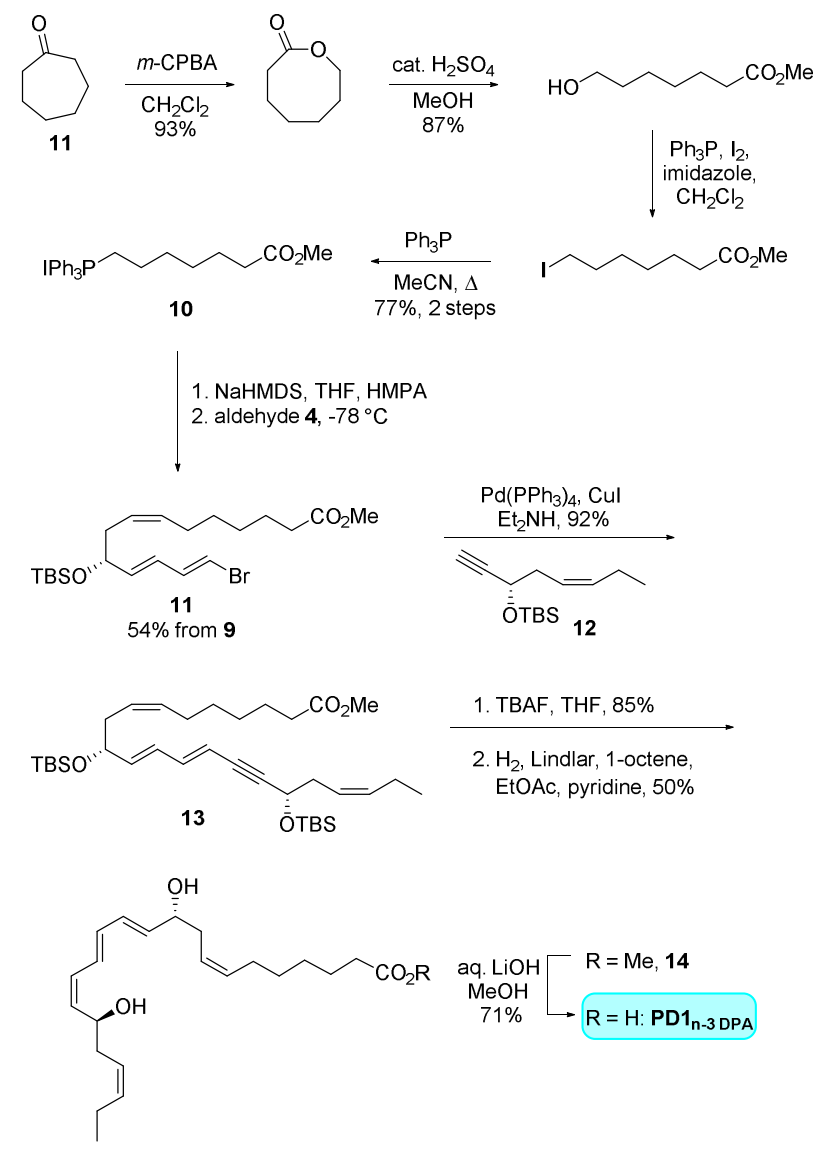

Scheme 3. The final steps in the synthesis of PD $1_{n-3}$ DPA.

The assignment of the $Z$ or $E$ geometry for each of the double bonds present in synthetic PD1 $1_{n-3}$ DPA was performed with the aid of two-dimensional NMR spectroscopy (COSY, HSQC and HMBC spectra). ${ }^{39}$ Matching studies using LC/MS-MS techniques confirmed that synthetic material of $P D 1_{n-3}$ DPA co-elutes with natural material obtained from murine selfresolving exudates and human macrophages. In addition, the MS/MS spectra for both natural and synthetic PD1 $1_{n-3}$ DPA were essentially identical. ${ }^{39}$ Overall, these efforts established the exact structure of $\mathrm{PD} 1_{\mathrm{n}-3}$ DPA to be $(7 Z, 10 R, 11 E, 13 E, 15 Z, 17 S, 19 Z)$-10,17-dihydroxydocosa7,11,13,15,19-pentaenoic acid.

Bioactions of $P D 1_{n-3 D P A}$ : Confirmation of the anti-inflammatory and pro-resolving actions of $\mathrm{PD} 1_{\mathrm{n}-3}$ DPA, in addition to matching of the physical properties described above, was an essential aspect of the matching process. ${ }^{39}$ Administration of a dose as low as $10 \mathrm{ng}$ per mouse significantly reduced neutrophil recruitment during peritonitis, giving a significant reduction in the number of neutrophils recovered from peritoneal exudates following Zymosan A challenge. The neutrophil count was determined by light microscopy and flow cytometry. These actions were comparable to those displayed by the potent DHA derived pro- 
resolving mediator protectin D1. ${ }^{39}$ Moreover, human macrophage phagocytosis and efferocytosis, as well as clearance of apoptotic neutrophils, are all important processes in the resolution of inflammation. These three key resolution mechanisms are defining actions of SPMs. ${ }^{6}$ Interestingly, when $\mathrm{PD} 1_{\mathrm{n}-3}$ DPA was incubated using increasing concentrations with human macrophages, a distinct increase in macrophage phagocytosis of both fluorescence labeled yeast cell wall particles (Zymosan A) as well as apoptotic neutrophils were observed. These actions were also comparable to those observed with protectin D1. Moreover, PD1 $1_{n-3}$ DPA also displayed potent efferocytosis effects with apoptotic human neutrophils, revealing a characteristic bell-shaped dose-response, with activities extending to the low nanomolar range. These findings demonstrated both potent anti-inflammatory and pro-resolving actions, confirming the potent immunoresolvent properties of $\mathrm{PD} 1_{\mathrm{n}-3 \mathrm{DPA}},{ }^{39}$ as discussed in the original report. $^{31}$

Isolation and structural elucidation MaR $1_{n-3} D P A$ : Since vascular leakage during inflammation supplies the site of inflammation with PUFAs and pro- and anti-inflammatory oxygenated mediators, Dalli et al. also investigated the tissue levels of n-3 DPA products during the onset and resolution of inflammation. ${ }^{31}$ For this purpose, a self-limited model of inflammation was used. Monitoring the administration of a pro-inflammatory stimulus into the mouse peritoneum, a time dependent recruitment of neutrophils was seen. Again, using targeted LC/MS-MS metabololipidomics analysis, three additional SPMs were detected compared to analyzing in vivo material obtained from reperfusion-induced injury. The three novel SPM compounds were named MaR $1_{n-3}$ DPA,$M a R 2_{n-3}$ DPA and $M a R 3_{n-3}$ DPA (see Figure 2 for structures), due to a shared alcohol at carbon 14 with maresin 1 . This particular experiment characterizes the initiation and resolution phases of the inflammatory response, in which the endogenous production of each individual SPM is observed in a time dependent manner that reflects their biosynthesis. The n-3 DPA product corresponding to MaR $1_{n-3}$ DPA gave levels that were elevated in the peritoneum of naive mice. After challenge with zymosan A, these levels were drastically decreased. It was also observed that MaR $1_{n-3}$ DPA accumulated later in the resolution processes, compared to PD1 $1_{\mathrm{n}-3}$ DPA as well as all other SPMs displayed in Figure 2. Again, combining data from UV-Vis spectroscopy and LC/MS-MS spectra, the chemical structure of MaR $1_{n-3}$ DPA was assigned to be $(7,8,10,12,14 S, 16 Z, 19 Z)$-7,14-dihydroxydocosa8,10,12,16,19-pentaenoic acid. ${ }^{31}$ The $S$-isomer of 14-HDPA, the reduction product of 14 $\mathrm{H} p$ DPA (15, Scheme 4), was identified as the major product in inflamed tissues. Therefore, it was reasoned to be likely that the stereochemistry at the 14-position is retained in the 
biosynthesis so that the configuration at C-14 in MaR $1_{n-3}$ DPA is in the $S$-configuration. ${ }^{31}$ The full configurational assignment of $\mathrm{MaR} 2_{\mathrm{n}-3 \mathrm{DPA}}$ and $\mathrm{MaR} 3_{\mathrm{n}-3}$ DPA has not yet been performed.

Biosynthesis of MaR $1_{n-3 D P A}$ : In a parallel pathway as mentioned for the biosynthesis of PD1 $1_{\mathrm{n}-3}$ DPA, the 12-LOX enzyme convert n-3 DPA to $14 S$-hydroperoxy-7Z,10Z,12E,16Z,19Zdocosapentaenoic acid (14-HpDPA, 15) that is further converted into $13,14 S$-epoxy $\mathrm{MaR}_{\mathrm{n}-3}$ DPA (16), which is enzymatically hydrolyzed to yield MaR $1_{n-3}$ DPA $(S c h e m e 4)$. An opening of 16 with water under enzymatically controlled conditions also provides MaR $2_{n-3}$ DPA. Recently it was disclosed that synthetic $13 S, 14 S$-epoxy-MaR was enzymatically converted to maresin 1 by human macrophages. ${ }^{47}$ It would be expected that the biosynthesis of maresin 1 and $M a R 1_{n-}$ 3 DPA, also two congeners, would occur in a similar fashion. Therefore, it is most likely that the stereochemical configuration of epoxide $\mathbf{1 6}$ is also $S, S$. However, conclusive evidence may be obtained be preparing synthetic $\mathbf{1 6}$ and conduct biological experiments and biosynthetic studies.

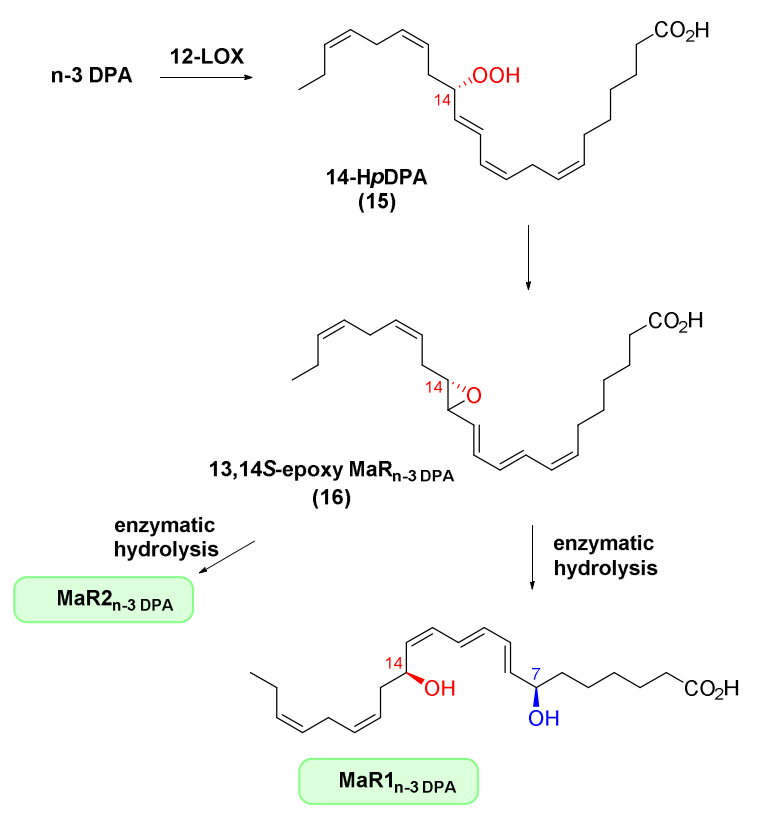

Scheme 4: Proposed biosynthesis of $M a R 1_{n-3}$ DPA

Total synthesis of MaR $1_{n-3}$ DPA: In 2014, Hansen and co-workers published the only reported synthesis of MaR $1_{n-3 \text { DPA. }}{ }^{48}$ Again, a convergent approach was chosen. The synthesis of the $\omega$ fragment 21 of MaR $1_{n-3}$ DPA began with the TBS-protection of commercially available $(S)-(-)-$ $\alpha$-hydroxy- $\gamma$-butyrolactone (17), followed by a reduction with DIBAL-H and a Colvin rearrangement to afford the primary alcohol 18. A Swern oxidation furnished silyl-protected pentynal 19 in $47 \%$ overall yield from 17. This aldehyde was then reacted in a $Z$-selective 
Wittig reaction with the ylide of salt $\mathbf{2 0}$, prepared in a direct manner over two steps from cis3-hexen-1-ol, to afford terminal alkyne 21. Formation of the unwanted $5 E$-isomer of 21 was suppressed by high dilution, low temperature and addition of hexamethylphosphoramide (HMPA). The undesired E-olefin was not detected after purification by column chromatography. Overall, the omega-end 21 was prepared in five steps starting from lactone $\mathbf{1 7}$ and in $39 \%$ overall yield.

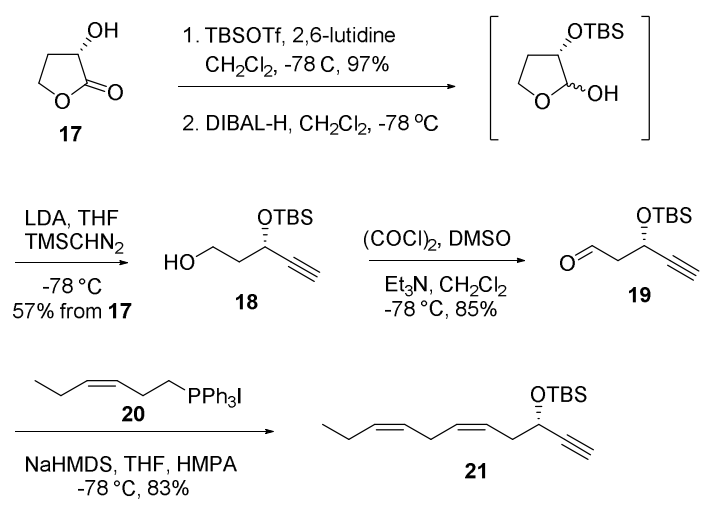

Scheme 5. Synthesis of fragment 21 for MaR $1_{n-3}$ DPA.

A Sonogashira cross-coupling reaction between the terminal alkyne $\mathbf{2 1}$ and vinyl bromide $\mathbf{2 2}$, the latter obtained by reductive cleavage of the chiral auxillary in 9 (prepared as previously shown in Scheme 2) with $\mathrm{LiBH}_{4}$, afforded the conjugated dienyne 23 in $68 \%$ yield from 22 (Scheme 6). The alcohol $\mathbf{2 3}$ was then converted to the corresponding alkyl bromide $\mathbf{2 4}$ using the Appel reaction.

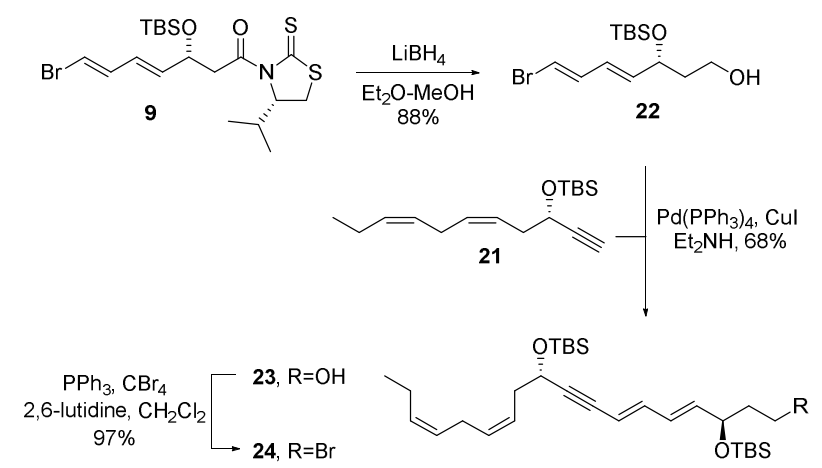

Scheme 6. Assembly of fragments 21 and 22 in the synthesis of MaR $1_{n-3}$ DPA.

The final key feature of this synthesis was a palladium catalyzed alkyl-alkyl $\mathrm{sp}^{3}$-sp $\mathrm{sp}^{3}$ coupling between bromide $\mathbf{2 4}$ and commercially available 4-ethoxy-4-oxobutylzinc bromide (25), see Scheme 7. A major challenge associated with alkyl substrates in palladium catalyzed crosscoupling reactions is the slow oxidative addition and the high probability of $\beta$-hydride 
elimination. Organ and co-workers have recently developed a Pd-based $N$-heterocycle carbene (NHC) pre-catalyst system which forms an electron rich and highly nucleophilic palladium species with significant steric bulk around the palladium center, thus limiting the competing $\beta$-hydride elimination. ${ }^{49,50}$ Moreover, the effectiveness of this reaction is also dependent on the addition of a lithium halide, which activate the alkyl zinc reagent through a lithium zincate. ${ }^{50}$ When alkyl bromide $\mathbf{2 4}$ and commercially available organozinc-reagent $\mathbf{2 5}$ were reacted in the presence of the Pd-PEPPSI-IPr catalyst $\mathbf{2 6}$ and $\mathrm{LiCl}$ in THF and NMP, a smooth cross coupling reaction occurred, yielding the coupled product 27 in 68\% yield (Scheme 7). As far as we know, this is by far the most complex substrate that has been applied to a PEPPSI catalyzed $\mathrm{sp}^{3}-\mathrm{sp}^{3}$ Negishi cross-coupling reaction. ${ }^{51,} 52$ This demonstrates the potential of this methodology, which may enable further access to complex natural products, including SPMs. With the construction of the whole carbon skeleton, only three steps remained for the completion of the total synthesis of MaR $1_{n-3}$ DPA. Deprotection of the two TBS-groups was achieved with TBAF in THF at $0{ }^{\circ} \mathrm{C}$, giving the corresponding diol in $97 \%$ yield. The chemo- and stereoselective semihydogenation was conducted with the modified Lindlar conditions. ${ }^{45,46}$ This afforded the ethyl ester of MaR $1_{n-3}$ DPA as a single isomer after purification by column chromatography and in $77 \%$ yield. Basic hydrolysis of the ethyl ester with lithium hydroxide completed the synthesis, yielding synthetic MaR $1_{n-3}$ DPA in $86 \%$ yield. Matching experiments using synthetic and authentic MaR $1_{n-3}$ DPA confirmed that the synthetic material displayed essentially identical physical properties to the biological material, suggesting that the structure of $\mathrm{MaR}_{\mathrm{n}-3}$ DPA was $(7 S, 8 E, 10 E, 12 Z, 14 S, 16 Z, 19 Z)-7,14-$ dihydroxydocosa-8,10,12,16,19-pentaenoic acid. ${ }^{48}$

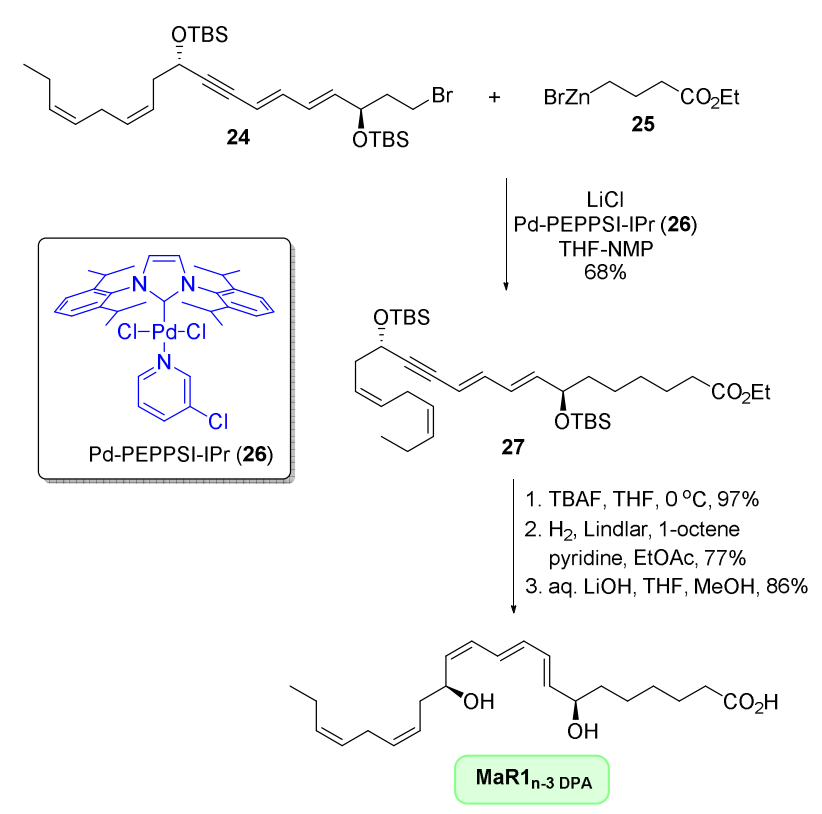


Scheme 7. End game in the synthesis of MaR $1_{n-3}$ DPA.

Bioactions of MaR $1_{n-3}$ DPA: Confirmation of the stereochemical configuration was obtained by matching the biological actions of the synthetic material with those reported for MaR $1_{n-3}$ DPA. For this purpose the ability of the synthetic material to activate macrophage responses was assessed. ${ }^{48}$ These studies revealed a very potent stimulation of macrophage efferocytosis of apoptotic human neutrophils, similar potency and activity to that of the DHA derived maresin 1. As of today, no in vivo studies have been reported using this SPM alone. However, a mixture of this SPM and MaR2 $2_{n-3}$ DPA significantly reduced PMN recruitment and proinflammatory cytokine exudate levels. ${ }^{31}$ Therefore one would anticipate that MaR $1_{n-3}$ DPA shares a biological profile similar to maresin 1 .

Isolation and structural elucidation of the 13-series resolvins: Neutrophil adherence to the vascular endothelium is a critical and early event in the innate immune response to invading pathogens. ${ }^{6}$ Very recently, when Dalli, Chiang and Serhan studied the lipid fractions from neutrophil-endothelial cell co-cultures, four novel oxygenated PUFA derivatives were discovered. ${ }^{53}$ These novel compounds were all derived from n-3 DPA with conjugated triene and diene double bonds present and a secondary alcohol at C-13 present in all four compounds. These n-3 DPA derived products were named 13-series resolvins (RvTs), or RvT1, RvT2, RvT3, and RvT4 due to the presence of a secondary alcohol in the 13-position for all compounds, see Figure 3.
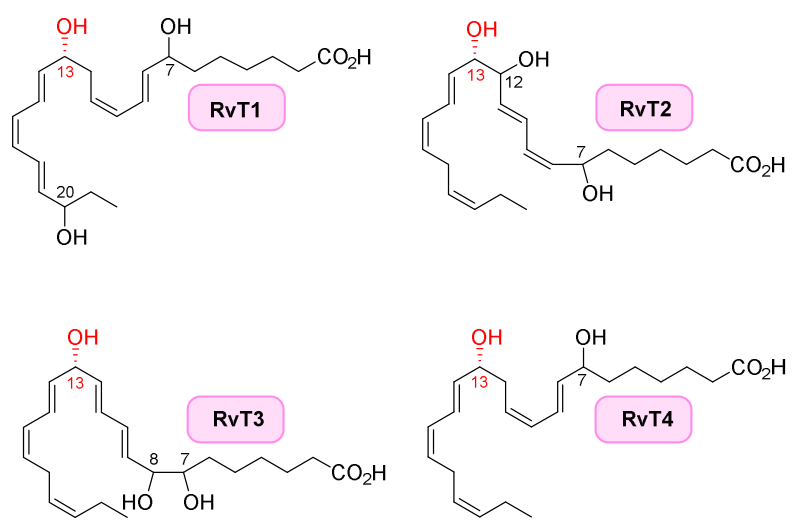

Figure 3. Chemical structures of RvT1 - RvT4. ${ }^{53}$

All four RvTs were rapidly formed ( $\leq 4$ hours) during the initial phase of inflammation in mice infected with E. coli. Moreover, RvT concentrations in peripheral blood increased rapidly after exercise, a common model used for a self-resolving inflammatory state, in healthy volunteers. Of note, the levels were significantly higher in patients with sepsis than in 
healthy persons. These data indicate that RvT biosynthesis occurs in a coordinated fashion upon acute activation of the immune response. Also for these novel compounds, LC/MS-MS based lipid mediator (LM) metabololipidomics was used for structural elucidations. The distinct MS-MS spectra of the four compounds gave characteristic signals for C22 containing compounds with five double bonds, suggesting that n-3 DPA was the precursor. The MS-MS fragmentations and UV-Vis-spectra aided the deduction of the structures as 7,13,20trihydroxy-docosapentaenoic acid (RvT1), 7,12,13-trihydroxy-docosapentaenoic acid (RvT2), 7,8,13-trihydroxy-docosapentaenoic acid (RvT3) and 7,13-dihydroxy-docosapentaenoic acid (RvT4). ${ }^{53}$ The structural assignments were also aided by the physical characteristics of different chemical derivatives for MS-MS-analysis and molecular oxygen $\left({ }^{18} \mathrm{O}_{2}\right)$ incorporation of each compound, as well as structural assignment of products derived from acid methanol trapping experiments. Co-incubations of neutrophil-endothelial cells with n-3 DPA increased their amounts to $30-50 \%$. Similar observations were not made using DHA or EPA as precursors to their distinct families of SPMs, rendering additional evidence for the direct biosynthesis from n-3 DPA into the 13-series resolvins. ${ }^{53}$ As of today, the exact configurational assignments of these interesting and novel resolvins have not been performed.

Biosynthetic studies towards RvT1 - RvT4 using synthetic 13R-HDPA: Dalli et al. reported that the RvT biosynthesis is a two-step transcellular process that requires neutrophilendothelial cell interactions. In the proposed biosynthesis of the 13-series resolvins, COX-2 converts n-3 DPA into 13R-hydroxy-7Z,10Z,14,16Z,19Z-docosapentaenoic acid (13R-HDPA) in endothelium cells. ${ }^{53}$ After transcellular trafficking to adjacent neutrophils, this intermediate is lipoxygenated by 5-LOX, producing RvT1-4 (see Scheme 8).

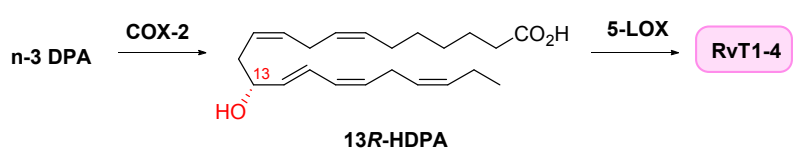

Scheme 8. Proposed biosynthesis of RvT1-4 via 13R-HDPA. ${ }^{53}$

Recently, evidence for the role of $13 R$-HDPA as an intermediate in the biosynthetic pathway towards all of the four RvTs was disclosed. ${ }^{54}$ After preparing synthetic 13R-HDPA, the compound was incubated with human neutrophils. This provided RvT1, RvT2, RvT3 and RvT4 as determined by LC/MS-MS experiments. Of note, in incubations with neutrophils without $13 R$-HDPA, the levels of these four lipid mediators were $>75 \%$ lower than those 
found in incubations with synthetic material. The preparation of synthetic 13R-HDPA was performed as outlined in Schemes 9-11. ${ }^{54}$ The omega end 28 of 13R-HDPA was prepared from the mesylate of 2-pentyn-1-ol (29) (Scheme 9). A copper mediated coupling with TMSacetylene 30 under basic conditions afforded diyne 31, which could be stereoselectively reduced to the $Z$-alkene 32. Removal of the TMS-group using TBAF buffered with acetic acid provided terminal alkyne 33. Compound $\mathbf{3 3}$ was then reacted in a Sonogashira cross-coupling reaction with vinyl iodide $\mathbf{3 4}$ to afford aldehyde $\mathbf{2 8}$ after oxidation of the corresponding alcohol with Dess-Martin periodinane.

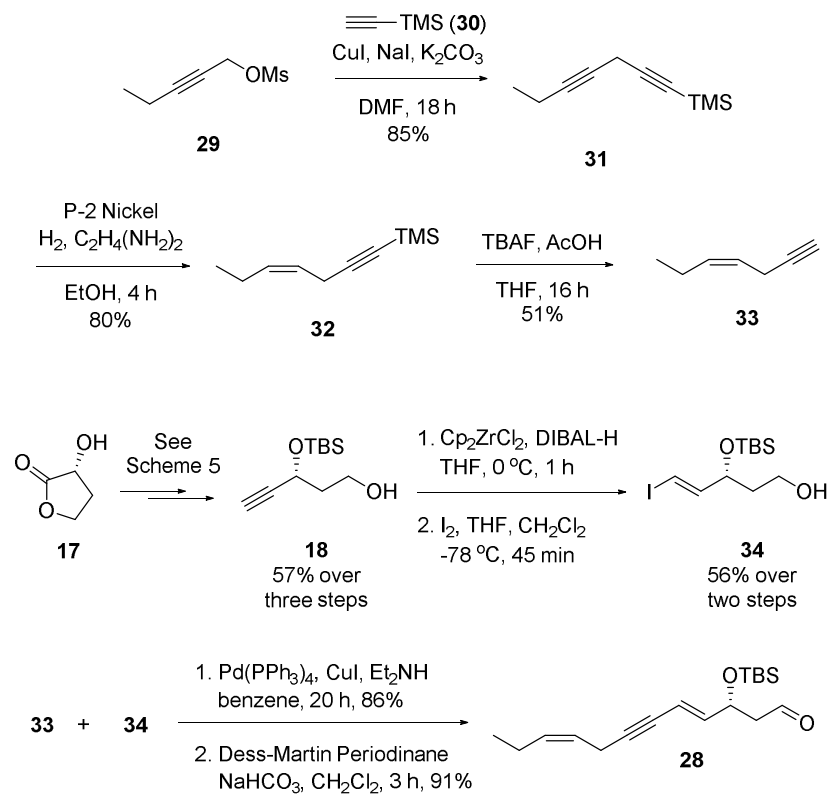

Scheme 9. Synthesis of the omega-end fragment 28.

The alpha-fragment 35 of $13 R$-HDPA (Scheme 10) was prepared from Wittig salt $10 .{ }^{39} \mathrm{~A}$ Wittig reaction between the ylide of $\mathbf{1 0}$ and TBS-protected 3-hydroxypropanal (36) yielded primary alcohol 37 after removal of the TBS-group. An Appel reaction followed by treatment with $\mathrm{PPh}_{3}$ in refluxing acetonitrile, afforded Wittig salt 35.

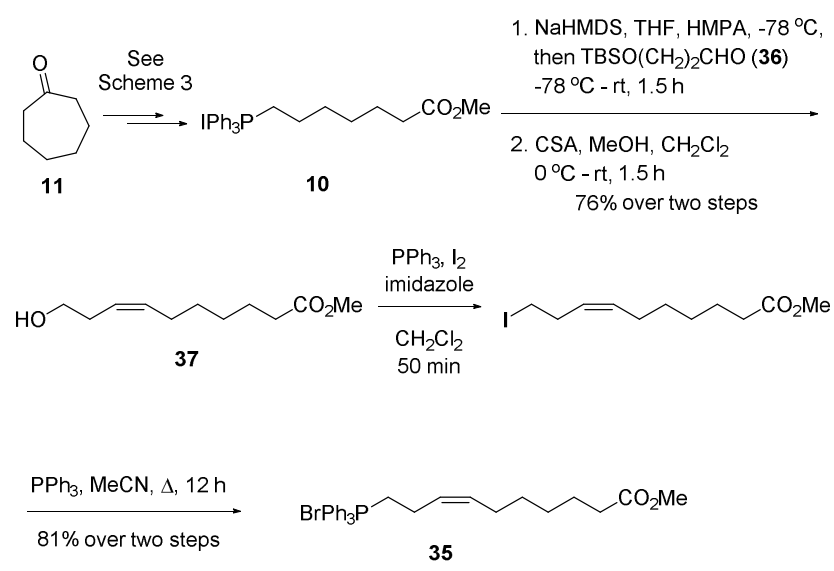


Scheme 10. Synthesis of the alpha-end fragment 35 .

In the end-game of the synthesis, aldehyde $\mathbf{2 8}$ and the ylide of Wittig salt $\mathbf{3 5}$ were reacted in a $Z$-selective Wittig reaction, affording the alkyne 38 in 77\% yield (Scheme11). After removal of the TBS-group with in situ generated $\mathrm{HCl}$ in methanol, the conjugated alkyne in $\mathbf{3 9}$ was stereoselectively reduced using the Boland protocol to afford the methyl ester of $13 R$-HDPA (40). Finally, basic hydrolysis with LiOH gave 13R-HDPA, which matched the authentic natural product. ${ }^{54}$

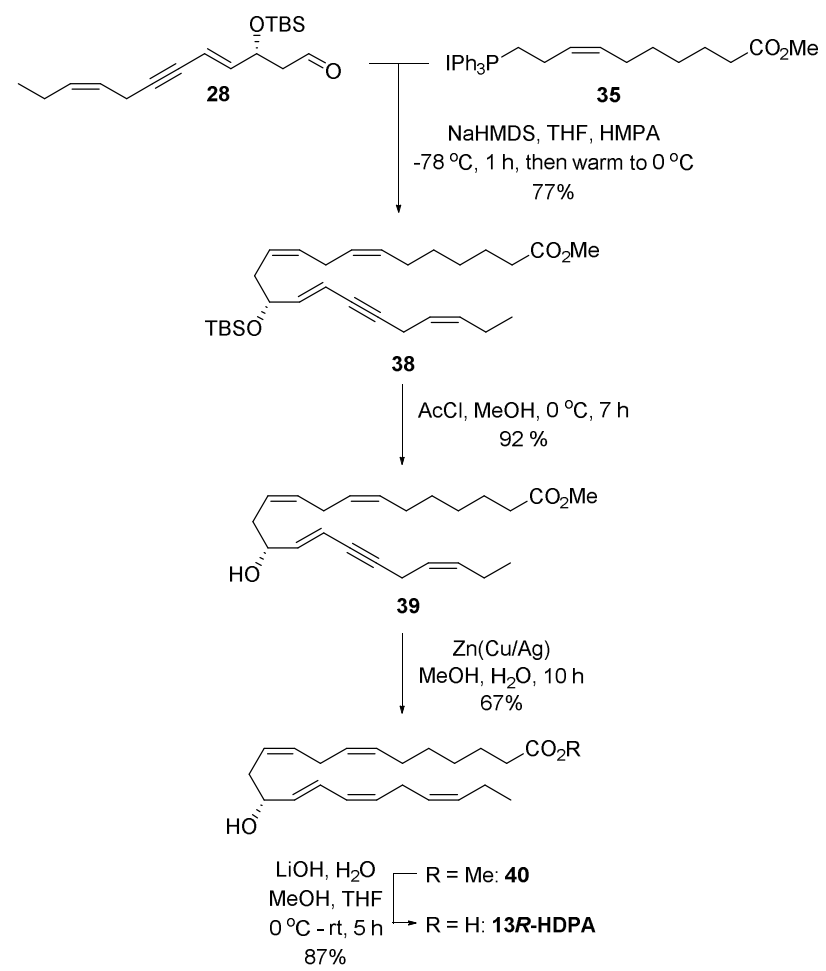

Scheme 11. The final stages in the synthesis of $13 R$-HDPA.

Further studies are needed to establish the individual steps and the detailed biosynthesis for each individual RvT. Evidence obtained using ${ }^{18} \mathrm{O}_{2}$ incorporation indicate the conversion of 13R-HDPA to RvT occurs via lipoxygenation reaction(s). In the biosynthesis of RvT2 and RvT3, acid alcohol trapping experiments suggest that the hydroperoxide formed via an initial lipoxygenation is then converted to an allylic epoxide, ${ }^{53}$ analogues to what is observed for leukotrienes, lipoxins, resolvins, protectins and maresis. ${ }^{36,47,55,56}$

Bioactions of RvT1-RvT4: RvT1 - RvT4 displayed several interesting biological activities when evaluated in infectious inflammation models. ${ }^{53}$ The administration of RvTs dosedependently promoted bacterial phagocytosis, and increased the amount of intracellular 
reactive oxygen species essential for bacterial killing, in isolated human neutrophils and macrophages. In addition an enhancement of the efferocytosis of apoptotic neutrophils was also observed, while the recruitment of neutrophils was dampened. Also, the activation of inflammasomes in macrophages was blocked with a concomitant reduction in levels of caspase 1 and interleukin-1 $\beta$. The vascular-endothelial system in humans is extensive. This, together with the abundance of neutrophils within this system, afforded information that the formation of RvTs during early stages of self-resolving acute inflammation could provide a molecular basis for the development of new treatment strategies for infectious inflammation. Since bacterial infections in humans remain a serious health concern due to the rise in antibiotic resistance, there is an urgent need for new strategies and development of new antibacterial drugs. ${ }^{57,58}$ Based on the bioactions mentioned above, RvTs are interesting lead compounds.

Conclusions and future perspectives: Resolution of inflammation is now held to be an active process where autacoids, including SPMs, promote homeostasis or the return to normal physiology. Using functional LC/MS-MS based metabololipidomics and predictive biological assays, several distinct families of SPMs biosynthesized from EPA, DHA and more recently n-3 DPA, have been isolated, their structures elucidated and subjected to total synthesis efforts. Total synthesis of SPMs has resulted in material available for further evaluations in several biological assays. Some SPMs have entered clinical trial development programs. ${ }^{28}$ Moreover, some synthetic analogs have been prepared and subjected to biological evaluations. $5,11,59,60$ We believe that these efforts will provide the basis for medicinal chemistry programs towards the development of novel anti-inflammatory drugs using immunoresolvents as biotemplates. In addition, this may also enable the development of new strategies for treating infections and chronic inflammatory diseases. However, such efforts will only be successful if the structure-function relationships are established, the biosynthetic pathway determined and the exact structural assignment for each individual SPM has been performed. Chemically pure n-3 DPA is now available by organic synthesis. ${ }^{61}$ The SPMs biosynthesized from this n-3 PUFA continue to attract interest from the biomedical community. ${ }^{62,63}$ We hope that the information outlined in this digest will be useful in medicinal chemistry efforts. Based on the rather high proportion of patients who do not respond to current anti-inflammatory therapies, the need for new, small molecular inflammatory drugs and immunoresolvents is evident. ${ }^{5,64-68}$ 


\section{Acknowledgements}

The Norwegian Research Council is gratefully acknowledged for generous funding to T.V.H (KOSK 197708, KOSK II 207053, BIOTEK2021 224811, FRIPRO-FRINATEK 230470). T.V.H. is also thankful for a Leiv Eriksson travel grant from The Norwegian Research Council enabling a sabbatical period with Professor Charles N. Serhan. J.D. received funding from the European Research Council (ERC) under the European Union's Horizon 2020 research and innovation programme (grant no: 677542) and the Barts Charity (grant no: MGU0343). J.D. is also supported by a Sir Henry Dale Fellowship jointly funded by the Wellcome Trust and the Royal Society (grant 107613/Z/15/Z).

\section{References}

1. G. Majno and I. Joris, Cells, tissues, and disease : principles of general pathology, Blackwell, Oxford, 2. edn., 2004, pages 307-382.

2. I. Tabas and C. K. Glass, Science, 2013, 339, 166-172.

3. B. Samuelsson, J. Biol. Chem., 2012, 287, 10070-10080.

4. C. N. Serhan and N. A. Petasis, Chem. Rev., 2011, 111, 5922-5943 and references cited therein.

5. C. N. Serhan, Nature, 2014, 510, 92-101.

6. C. N. Serhan, Annu. Rev. Immunol., 2007, 25, 101-137.

7. C. N. Serhan, M. Hamberg and B. Samuelsson, Biochem. Biophys. Res. Commun., 1984, 118, 943-949.

8. C. N. Serhan, M. Hamberg and B. Samuelsson, Proc. Natl. Acad. Sci. U. S. A., 1984, 81, 5335-5339.

9. B. D. Levy, Prostaglandins Leukot. Essent. Fatty Acids, 2005, 73, 231-237.

10. M. Romano, E. Cianci, F. Simiele and A. Recchiuti, Eur. J. Pharmacol., 2015, 760, 49-63.

11. N. A. Petasis, I. Akritopoulou-Zanze, V. V. Fokin, G. Bernasconi, R. Keledjian, R. Yang, J. Uddin, K. C. Nagulapalli and C. N. Serhan, Prostaglandins Leukot. Essent. Fatty Acids, 2005, 73, 301-321.

12. C. N. Serhan, C. B. Clish, J. Brannon, S. P. Colgan, N. Chiang and K. Gronert, J. Exp. Med., 2000, 192, 1197-1204.

13. C. N. Serhan, S. Hong, K. Gronert, S. P. Colgan, P. R. Devchand, G. Mirick and R.-L. Moussignac, J. Exp. Med., 2002, 196, 1025-1037.

14. S. Hong, K. Gronert, P. R. Devchand, R.-L. Moussignac and C. N. Serhan, J. Biol. Chem., 2003, 278, 14677-14687. 
15. P. K. Mukherjee, V. L. Marcheselli, C. N. Serhan and N. G. Bazan, Proc. Natl. Acad. Sci. U. S. A., 2004, 101, 8491-8496.

16. C. N. Serhan, R. Yang, K. Martinod, K. Kasuga, P. S. Pillai, T. F. Porter, S. F. Oh and M. Spite, J. Exp. Med., 2009, 206, 15-23.

17. J. Dalli, S. Ramon, P. C. Norris, R. A. Colas and C. N. Serhan, FASEB J., 2015, 29, 21202136.

18. S. Ramon, J. Dalli, J. M. Sanger, J. W. Winkler, M. Aursnes, J. E. Tungen, T. V. Hansen and C. N. Serhan, Am. J. Pathol., 2016, 186, 962-973.

19. J. Dalli, R. A. Colas, H. Arnardottir and C. N. Serhan, Immunity, 2017, 46, 92-105.

20. A. R. Rodriguez and B. W. Spur, Tetrahedron Lett., 2015, 56, 5811-5815.

21. J. Dalli, N. Chiang and C. N. Serhan, Proc. Natl. Acad. Sci. U. S. A., 2014, 111, E4753-E4761.

22. J. Dalli, J. M. Sanger, A. R. Rodriguez, N. Chiang, B. W. Spur and C. N. Serhan, PLoS One, 2016, 11, e0149319/0149311-e0149319/0149312.

23. J. Dalli, I. Vlasakov, I. R. Riley, A. R. Rodriguez, B. W. Spur, N. A. Petasis, N. Chiang and C. N. Serhan, Proc. Natl. Acad. Sci. U. S. A., 2016, 113, 12232-12237.

24. A. R. Rodriguez and B. W. Spur, Tetrahedron Lett., 2015, 56, 3936-3940.

25. A. R. Rodriguez and B. W. Spur, Tetrahedron Lett., 2017, DOI: http://dx.doi.org/10.1016/j.tetlet.2017.03.041.

26. N. Chiang, G. Fredman, F. Baeckhed, S. F. Oh, T. Vickery, B. A. Schmidt and C. N. Serhan, Nature, 2012, 484, 524-528.

27. C. N. Serhan, J. Dalli, R. A. Colas, J. W. Winkler and N. Chiang, Biochim. Biophys. Acta, Mol. Cell Biol. Lipids, 2015, 1851, 397-413.

28. C. N. Serhan and N. Chiang, Br. J. Pharmacol., 2008, 153, S200-S215.

29. M. G. Duvall and B. D. Levy, Eur. J. Pharmacol., 2016, 785, 144-155.

30. C. N. Serhan and N. Chiang, Curr. Opin. Pharmacol., 2013, 13, 632-640.

31. J. Dalli, R. A. Colas and C. N. Serhan, Sci. Rep., 2013, 3, 1940.

32. S. Ferdinandusse, S. Denis, P. A. W. Mooijer, Z. Zhang, J. K. Reddy, A. A. Spector and R. J. A. Wanders, J. Lipid Res., 2001, 42, 1987-1995.

33. G. Kaur, D. Cameron-Smith, M. Garg and A. J. Sinclair, Prog. Lipid Res., 2011, 50, 28-34.

34. J. Z. Haeggström and C. D. Funk, Chem. Rev., 2011, 111, 5866-5898.

35. C. N. Serhan, K. Gotlinger, S. Hong, Y. Lu, J. Siegelman, T. Baer, R. Yang, S. P. Colgan and N. A. Petasis, J. Immunol., 2006, 176, 1848-1859.

36. M. Aursnes, J. E. Tungen, R. A. Colas, I. Vlasakov, J. Dalli, C. N. Serhan and T. V. Hansen, J. Nat. Prod., 2015, 78, 2924-2931.

37. S. C. N., K. Gotlinger, S. Hong, Y. Lu, J. Siegelman, T. Baer, R. Yang, S. P. Colgan and N. A. Petasis, J. Immunology, 2006, 176, 1848-1559. 
38. A. Vik and T. V. Hansen, in From Biosynthesis to Total synthesis, ed. A. L. Zografos, Wiley, New Jersey, 2016, pp. 130-161.

39. M. Aursnes, J. E. Tungen, A. Vik, R. Colas, C.-Y. C. Cheng, J. Dalli, C. N. Serhan and T. V. Hansen, J. Nat. Prod., 2014, 77, 910-916.

40. J. M. J. Nolsøe, M. Aursnes, J. E. Tungen and T. V. Hansen, J. Org. Chem., 2015, 80, 53775385 .

41. Y. Nagao, W. M. Dai, M. Ochiai and M. Shiro, J. Org. Chem., 1989, 54, 5211-5217.

42. R. Tello-Aburto, A. Ochoa-Teran and H. F. Olivo, Tetrahedron Lett., 2006, 47, 5915-5917.

43. M. Romero-Ortega, D. A. Colby and H. F. Olivo, Tetrahedron Lett., 2002, 43, 6439-6441.

44. M. Aursnes, J. E. Tungen, A. Vik, J. Dalli and T. V. Hansen, Org. Biomol. Chem., 2014, 12, 432-437.

45. T. L. Ho and S. H. Liu, Synthetic. Commun., 1987, 17, 969-973.

46. J. Chang and L. A. Paquette, Org. Lett., 2002, 4, 253-256.

47. J. Dalli, M. Zhu, N. A. Vlasenko, B. Deng, J. Z. Haeggström, N. A. Petasis and C. N. Serhan, FASEB J., 2013, 27, 2573-2583, 2510.1096/fj.2513-227728.

48. J. E. Tungen, M. Aursnes, J. Dalli, H. Arnardottir, C. N. Serhan and T. V. Hansen, Chem. Eur. J., 2014, 20, 14575-14578.

49. M. Pompeo, R. D. J. Froese, N. Hadei and M. G. Organ, Angew. Chem., Int. Ed., 2012, 51, 11354-11357.

50. M. G. Organ, S. Avola, I. Dubovyk, N. Hadei, E. A. B. Kantchev, C. J. O'Brien and C. Valente, Chem. - Eur. J., 2006, 12, 4749-4755.

51. E. Geist, A. Kirschning and T. Schmidt, Nat. Prod. Rep., 2014, 31, 441-448.

52. D. Haas, J. M. Hammann, R. Greiner and P. Knochel, ACS Catal., 2016, 6, 1540-1552.

53. J. Dalli, N. Chiang and C. N. Serhan, Nat. Med., 2015, 21, 1071-1075.

54. K. G. Primdahl, M. Aursnes, M. E. Walker, R. A. Colas, C. N. Serhan, J. Dalli, T. V. Hansen and A. Vik, J. Nat. Prod., 2016, 79, 2693-2702.

55. Y.-P. Sun, S. F. Oh, J. Uddin, R. Yang, K. Gotlinger, E. Campbell, S. P. Colgan, N. A. Petasis and C. N. Serhan, J. Biol. Chem., 2007, 282, 9323-9334.

56. J. W. Winkler, J. Uddin, C. N. Serhan and N. A. Petasis, Org. Lett., 2013, 15, 1424-1427.

57. H. C. Neu, Science, 1992, 257, 1064-1072.

58. P. A. Ward, EMBO Mol. Med., 2012, 4, 1234-1243.

59. S. K. Orr, R. A. Colas, J. Dalli, N. Chiang and C. N. Serhan, Am. J. Physiol., 2015, 308, 1-8.

60. L. V. Norling, M. Spite, R. Yang, R. J. Flower, M. Perretti and C. N. Serhan, J. Immunology, 2011, 186, 5543-5547.

61. M. G. Jakobsen, A. Vik and T. V. Hansen, Tetrahedron Lett., 2012, 53, 5837-5839.

62. C. R. Lee and D. C. Zeldin, N. Engl. J. Med., 2015, 373, 2183-2185. 
63. T. Gobbetti, J. Dalli, R. A. Colas, D. F. Canova, M. Aursnes, D. Bonnet, L. Alric, N. Vergnolle, C. Deraison, T. V. Hansen, C. N. Serhan and M. Perretti, Proc. Natl. Acad. Sci., 2017, In press.

64. O. Corminboeuf and X. Leroy, J. Med. Chem., 2015, 58, 537-559.

65. M. C. Basil and B. D. Levy, Nat. Rev. Immunol., 2016, 16, 51-67.

66. M. Perretti, D. Cooper, J. Dalli and L. V. Norling, Nat. Rev. Rheumatol., 2017, 13, 87-99.

67. M. Perretti, X. Leroy, E. J. Bland and T. Montero-Melendez, Trends Pharmacol. Sci., 2015, 36, 737-755.

68. J. N. Fullerton and D. W. Gilroy, Nat. Rev. Drug Discovery, 2016, 15, 551-567. 